\title{
Mice further research on hearing loss
}

Research groups studying different types of hearing loss have gained new insight into its causes and possible treatments from experiments using mice. One group, led by Zheng-Yi Chen (Harvard Medical School and Massachusetts Eye and Ear Infirmary, Boston, MA), investigated agerelated hearing loss (ARHL) and noiseinduced hearing loss (NIHL), the two most common forms of deafness. Both are caused by the irreversible loss of inner ear outer hair cells, and although hearing aids and cochlear implants can help some affected individuals, they do not work for everyone, and there is no cure. Chen's team hypothesized that ARHL and NIHL might have similar causes and set out to determine whether protecting hair cells from damage could reduce either form of hearing loss. They zeroed in on the transcription factor islet 1 (Isl1) as a potential protector because it is expressed in developing hair cells. They created transgenic mice overexpressing Isl 1 only in hair cells and evaluated their hair cells and hearing function as they aged to
17 months. Isl1 overexpression protected hair cells from age-related degeneration and promoted hair cell survival after exposure to loud noise. As a result, transgenic mice had better hearing than wild-type mice of similar age or with similar exposure to loud noise (J. Neurosci. 33, 15086-15094; 2013). These results suggest that ARHL and NIHL likely share a common mechanism and, furthermore, that Isll expression can protect against the hearing loss that occurs with age and noise exposure.

A separate team headed by Kerry A. Miller and based at Murdoch Childrens Research Institute and University of Melbourne (Melbourne, Australia) approached the problem of hearing loss from a different angle. As part of a large-scale mutagenesis screen, they created mice with severe hearing loss, located the genetic region that was affected and characterized the condition as a model for human hereditary deafness. Their objective was to identify deafnessrelated genes that could become therapeutic targets for hereditary hearing loss in people.

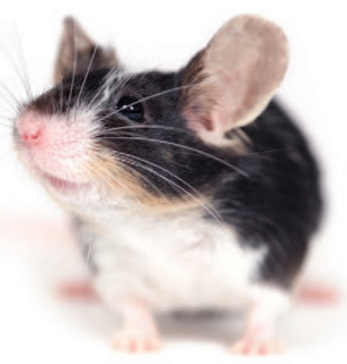

The mutant mice developed congenital hearing loss by 4 weeks of age, progressing to profound deafness within the next 8 weeks. They had abnormal cochlear structure and morphology. The causative mutation was located within a 21.6-Mb region of chromosome 18 that has not previously been associated with deafness but contains several candidates for further analysis, including the genes encoding aquaporin 4 , cadherin 2 and myosin 7b (PLoS ONE 8, e74243; 2013).

Both groups hope their work will lead to better understanding and treatment of hearing loss in humans.

Monica Harrington

\section{ADOLESCENT CAFFEINE CONSUMPTION SLOWS BRAIN DEVELOPMENT}

Adolescence is a critical period for brain maturation involving a massive reorganization of cortical connectivity. An overproduction of cortical synapses during early development leads to a peak in synaptic density shortly before puberty, followed by the selective elimination of synapses during adolescence. This process is essential for optimizing the brain's connections to make it function more efficiently.

Slow wave activity (SWA) during non-rapid eye movement sleep has been proposed to reflect cortical maturation. An increase in the amplitude of the slow waves is associated with a more synchronous firing pattern among neurons. This synchronicity increases in pre-pubertal children, reaches a maximum at puberty and decreases during adolescence, mirroring the inverted U-shaped trajectory of synapse density.

Caffeine, whose consumption by adolescents has recently been increasing, is known to diminish the buildup of sleep pressure during wakefulness. Because SWA is the major electrophysiological marker of sleep pressure, Reto Huber (University Children's Hospital Zurich, Switzerland) hypothesized that caffeine might interfere with SWA, and thus the changes in synaptic density necessary for brain maturation.

Huber's team assessed sleep, behavior and electrophysiological markers of brain maturation in the juvenile rat (PLOS ONE 8, e72539; 2013). As in humans, SWA in the rat increased progressively during early development, reached a plateau during puberty and declined significantly afterward. The number of cortical synapses also declined with increased age after puberty. These changes were paralleled by behavioral changes assessed in a free exploration task: more mature animals showed increased exploration of a novel object. These findings confirmed that the rat serves as an appropriate model of the relationship between SWA during sleep and cortical maturation.

They next used this rat model to determine the effects of caffeine consumption in adolescence. After the rats reached puberty, one group was administered caffeine in their drinking water for 5 days. The caffeine-treated rats exhibited higher SWA over the next few days when compared to the non-treated rats, and the reduction in the number of synapses seen in the non-treated rats was diminished. Furthermore, the age-dependent increase in exploration seen in the non-treated rats was reduced in the caffeine-treated rats.

These findings suggest that caffeine consumption delays cortical maturation during a critical developmental period by interfering with SWA during sleep. Because the critical period of synapse elimination during adolescence is associated with psychiatric and mood disorders, increased caffeine consumption in adolescents may be linked to the higher incidences of schizophrenia and personality disorders in recent years. 